PROCEEDINGS OF THE

AMERICAN MATHEMATICAL SOCIETY

Volume 131, Number 12, Pages 3695-3702

S 0002-9939(03)07216-2

Article electronically published on July 17, 2003

\title{
FINER GEOMETRIC RIGIDITY OF LIMIT SETS OF CONFORMAL IFS
}

\author{
VOLKER MAYER AND MARIUSZ URBAŃSKI
}

(Communicated by Michael Handel)

\begin{abstract}
We consider infinite conformal iterated function systems in the phase space $\mathbb{R}^{d}$ with $d \geq 3$. Let $J$ be the limit set of such a system. Under a mild technical assumption, which is always satisfied if the system is finite, we prove that either the Hausdorff dimension of $J$ exceeds the topological dimension $k$ of the closure of $J$ or else the closure of $J$ is a proper compact subset of either a geometric sphere or an affine subspace of dimension $k$. A similar dichotomy holds for conformal expanding repellers.
\end{abstract}

\section{INTRODUCTION AND PRELIMINARIES}

In this paper we explore the finer geometric structure of limit sets $J$ of infinite conformal iterated function systems. Under a natural easily verifiable technical condition (always satisfied if the system is finite), we demonstrate the following dichotomy. Either the Hausdorff dimension of $J$ exceeds the topological dimension $k$ of the closure of $J$ or else the closure of $J$ is a proper compact subset of either a geometric sphere or an affine subspace of dimension $k$. (In addition, if any one of our conformal contracting mappings is a similarity, then the latter case holds.) This is a strengthening of the following weaker dichotomy proven in MMU]: if $\bar{J}$ is connected, then either the Hausdorff dimension of $J$ exceeds 1 or else $\bar{J}$ is a proper compact segment of either a geometric circle or a straight line. The picture is now much clearer. We would like to add that so far, up to our knowledge, all the similar works concerned the same dichotomy as that produced in MMU (see, for example, ([Bo], [MU2], [Ma1, [ $\mathrm{Pr},[\mathrm{Ru},[\mathrm{Su}],[\mathrm{U} 1],[\mathrm{UV}],[\mathrm{Z1}],[\mathrm{Z} 2]$ ) most of which deal with the plane case $d=2$ and apply the Riemann Mapping Theorem. Our approach is based on an extensive use of the concept of rectifiablity.

To start the preliminaries concerning conformal iterated function systems, let $I$ be a countable index set with at least two elements and let $S=\left\{\phi_{i}: X \rightarrow\right.$ $X: i \in I\}$ be a collection of injective contractions from a compact set $\mathrm{X}$ into $\mathrm{X}$ for which there exists $0<s<1$ such that $\rho\left(\phi_{i}(x), \phi_{i}(y)\right) \leq s \rho(x, y)$ for every $i \in I$ and for every pair of points $x, y \in X$. Thus, the system $S$ is uniformly contractive. Any such collection $S$ of contractions is called an iterated function system. We are particularly interested in the properties of the limit set defined by such a system. We define this set as the image of the coding space under a

Received by the editors November 18, 2001.

2000 Mathematics Subject Classification. Primary 37D45, 37D20, 28Exx.

The second author was supported in part by the NSF Grant no. DMS 0100078.

(C)2003 American Mathematical Society 
coding map as follows. Let $I^{*}=\bigcup_{n \geq 1} I^{n}$, the space of finite words, and for $\tau \in I^{n}$, $n \geq 1$, let $\phi_{\tau}=\phi_{\tau_{1}} \circ \phi_{\tau_{2}} \circ \cdots \circ \phi_{\tau_{n}}$. Let $I^{\infty}=\left\{\left\{\tau_{n}\right\}_{n=1}^{\infty}\right\}$ be the set of all infinite sequences of elements of $I$. If $\tau \in I^{*} \cup I^{\infty}$ and $n \geq 1$ does not exceed the length of $\tau$, we denote by $\left.\tau\right|_{n}$ the word $\tau_{1} \tau_{2} \ldots \tau_{n}$. Since given $\tau \in I^{\infty}$, the diameters of the compact sets $\phi_{\left.\tau\right|_{n}}(X), n \geq 1$, converge to zero and since they form a descending family, the set

$$
\bigcap_{n=0}^{\infty} \phi_{\left.\tau\right|_{n}}(X)
$$

is a singleton and therefore, denoting its only element by $\pi(\tau)$, we define the coding map

$$
\pi: I^{\infty} \rightarrow X
$$

The main object in the theory of iterated function systems is the limit set defined as follows:

$$
J=\pi\left(I^{\infty}\right)=\bigcup_{\tau \in I^{\infty}} \bigcap_{n=1}^{\infty} \phi_{\tau \mid n}(X) .
$$

Observe that $J$ satisfies the natural invariance equality, $J=\bigcup_{i \in I} \phi_{i}(J)$. Notice that if $I$ is finite, then $J$ is compact and this property fails for infinite systems. Let $S(\infty)$ be the set of limit points of all sequences $x_{i} \in \phi_{i}(X), i \in I^{\prime}$, where $I^{\prime}$ ranges over all infinite subsets of $I$. In [MU1] the following has been proved.

Proposition 1.1. If $\lim _{i \in I} \operatorname{diam}\left(\phi_{i}(X)\right)=0$, then $\bar{J}=J \cup \bigcup_{\omega \in I^{*}} \phi_{\omega}(S(\infty))$.

An iterated function system $S$ is said to be conformal if $X \subset \mathbb{R}^{d}$ for some $d \geq 1$ and the following conditions are satisfied.

(1a): Open Set Condition (OSC). $\phi_{i}(\operatorname{Int} X) \cap \phi_{j}(\operatorname{Int} X)=\emptyset$ for every pair $i, j \in I, i \neq j$.

(1b): There exists an open connected set $V$ such that $X \subset V \subset \mathbb{R}^{d}$ and such that all maps $\phi_{i}, i \in I$, extend to $C^{1}$ conformal diffeomorphisms of $V$ into $V$. (Note that for $d=1$ this just means that all the maps $\phi_{i}, i \in I$, are $C^{1}$ monotone diffeomorphisms, for $d \geq 2$ the word conformal means holomorphic or antiholomorphic, and for $d \geq 3$, the maps $\phi_{i}, i \in I$ are Möbius transformations. (The proof of the last statement can be found in $\mathrm{BP}$ and $\mathrm{Va}$, for example, where it is called Liouville's theorem.)

(1c): (Cone Condition) There exist $\alpha, l>0$ such that for every $x \in \partial X \subset$ $\mathbb{R}^{d}$ there exists an open cone $\operatorname{Con}(x, u, \alpha) \subset \operatorname{Int}(X)$ with vertex $x$, the symmetry axis determined by vector $u \in \mathbb{R}^{d}$ of length $l$, and a central angle of Lebesgue measure $\alpha$. Here $\operatorname{Con}(x, u, \alpha, l)=\{y: 0<(y-x, u) \leq$ $\cos \alpha\|y-x\| \leq l\}$.

(1d): (Bounded Distortion Property (BDP)). There exists $K \geq 1$ such that

$$
\left|\phi_{\tau}^{\prime}(y)\right| \leq K\left|\phi_{\tau}^{\prime}(x)\right|
$$

for every $\tau \in I^{*}$ and every pair of points $x, y \in V$, where $\left|\phi_{\tau}^{\prime}(x)\right|$ means the norm of the derivative.

Under these assumptions it was shown in MU1 that the hypothesis of Proposition 1.1 holds and we can change the order of the union and intersection operations 
to obtain:

$$
J=\pi\left(I^{\infty}\right)=\bigcap_{n \geq 1} \bigcup_{|\tau|=n} \phi_{\tau}(X)
$$

Let us first collect some geometric consequences of (BDP). We have for all words $\tau \in I^{*}$ and all convex subsets $C$ of $V$,

$$
\operatorname{diam}\left(\phi_{\tau}(C)\right) \leq\left\|\phi_{\tau}^{\prime}\right\| \operatorname{diam}(C)
$$

and

$$
\operatorname{diam}\left(\phi_{\tau}(V)\right) \leq D\left\|\phi_{\tau}^{\prime}\right\|,
$$

where the norm $\|\cdot\|$ is the supremum norm taken over $V$ and $D \geq 1$ is a universal constant. Moreover,

$$
\operatorname{diam}\left(\phi_{\tau}(J)\right) \geq D^{-1}\left\|\phi_{\tau}^{\prime}\right\|
$$

and

$$
\phi_{\tau}(B(x, r)) \supset B\left(\phi_{\tau}(x), K^{-1}\left\|\phi_{\tau}^{\prime}\right\| r\right),
$$

for every $x \in X$, every $0<r \leq \operatorname{dist}(X, \partial V)$, and every word $\tau \in I^{*}$.

From now on throughout the entire paper we assume that $d \geq 2$. By $\mathrm{H}^{t}$ we will denote the $t$-dimensional Hausdorff measure, by HD the Hausdorff dimension and by TD the topological dimension (we will only deal with subsets of $\mathbb{R}^{d}$ so that all Hausdorff and topological dimensions are finite). The main result of our paper is the following.

Theorem 1.2. If $d \geq 3, S=\left\{\phi_{i}\right\}_{i \in I}$ is a conformal IFS and $\operatorname{HD}(S(\infty))<\operatorname{HD}(J)$, then either

(a): $\operatorname{HD}(J)>\operatorname{TD}(\bar{J})$ or

(b): $\bar{J}$ is a proper compact subset of either a geometric sphere of dimension $\operatorname{TD}(\bar{J})$ or a $\operatorname{TD}(\bar{J})$-dimensional affine hyperspace, both contained in $\mathbb{R}^{d}$.

In addition, if any one of the maps $\phi_{i}$ is a similarity mapping, then the latter case holds.

Since in the finite case the set $S(\infty)$ is empty, we get immediately the following.

Corollary 1.3. If $d \geq 3, S=\left\{\phi_{i}\right\}_{i \in I}$ is a finite conformal IFS, then either

(a): $\operatorname{HD}(J)>\operatorname{TD}(\bar{J})$ or

(b): $\bar{J}$ is a proper compact subset of either a geometric sphere of dimension $\operatorname{TD}(\bar{J})$ or a $\operatorname{TD}(\bar{J})$-dimensional affine hyperspace, both contained in $\mathbb{R}^{d}$.

In addition, if any one of the maps $\phi_{i}$ is a similarity mapping, then the latter case holds.

Remark 1.4. Put $k=\operatorname{TD}(\bar{J})$. Since a compact subset of a $k$-dimensional sphere or hyperspace $G$ has topological dimension $k$ if and only if its interior in $G$ is not empty, we see that in the second alternative of Theorem 1.2 and Corollary $1.3, \bar{J}$ contains an open ball in the appropriate sphere or hyperspace and, for dynamical reasons, it turns out that there is an open subset $\Omega$ of that sphere or hyperspace such that $\bar{J}=\bar{\Omega}$.

With essentially the same methods as those employed in the proof of Theorem 1.2 one can prove the following. 
Theorem 1.5. If $F: Y \rightarrow Y$ is a conformal expanding repeller, then the same dichotomy holds as in Corollary 1.3. Only one geometric sphere or an affine hyperspace is to be replaced by a finite union of such sets. In the case when $Y$ is connected this finite union also reduces to either one sphere or one hyperspace.

Let us now recall from MU1 that a Borel probability measure $m$ is said to be $t$-conformal provided $m(J)=1$ and for every Borel set $A \subset X$ and every $i \in I$,

$$
m\left(\phi_{i}(A)\right)=\int_{A}\left|\phi_{i}^{\prime}\right|^{t} d m
$$

and

$$
m\left(\phi_{i}(X) \cap \phi_{j}(X)\right)=0,
$$

for every pair $i, j \in I, i \neq j$. It has been proved in [MU1] that if a $t$-conformal measure exists, then $t=h$, the Hausdorff dimension of the limit set $J$ of $S$, and this measure is unique. The system $S$ is called regular if a conformal measure exists. Let $\sigma: \Sigma \rightarrow \Sigma$ be the left shift transformation (cutting out the first coordinate), $\sigma\left(\left\{x_{n}\right\}_{n=1}^{\infty}\right)=\left(\left\{x_{n}\right\}_{n=2}^{\infty}\right)$. In [MU1] we have proved the following.

Theorem 1.6. If the system $S$ is regular and $m$ is the $h$-conformal measure, then

(a): There exists a unique Borel probability measure $\tilde{m}$ on $I^{\infty}$ such that $\tilde{m} \circ$ $\pi^{-1}=m$. The measure $\tilde{m}$ is positive on nonempty open subsets of $I^{\infty}$.

(b): There exists a unique $\sigma$-invariant probability measure $\tilde{\mu}$ absolutely continuous with respect to $\tilde{m}$. Moreover, $\tilde{\mu}$ is equivalent with $\tilde{m}, \sup \left\{\left|\log \left(\frac{d \tilde{\mu}}{d \tilde{m}}\right)\right|\right\}$ $<\infty$ and the dynamical system $\sigma: I^{\infty} \rightarrow I^{\infty}$ is ergodic with respect to the measure $\tilde{\mu}$.

\section{ReCtifiability and TANGENTS}

A set $Q \subset \mathbb{R}^{d}$ is called $k$-rectifiable if $\mathrm{H}^{k}(Q)>0$ and there exist Lipschitz maps $g_{i}: \mathbb{R}^{k} \rightarrow \mathbb{R}^{d}, i=1,2, \ldots$ such that

$$
\mathrm{H}^{k}\left(Q \backslash \bigcup_{i=1}^{\infty} g_{i}\left(\mathbb{R}^{k}\right)\right)=0 .
$$

A set $T \subset \mathbb{R}^{d}$ is called purely $k$-unrectifiable if and only if $\mathrm{H}^{k}(Q \cap T)=0$ for every $k$-rectifiable set $Q$.

It follows from Theorem 15.19 in $\mathrm{Ma2}$ that for $\mathrm{H}^{k}$-a.e. point $z$ in a $k$-rectifiable set $Q \subset \mathbb{R}^{d}$ there is a unique approximate tangent $k$-plane for $Q$ at $z$. This tangent plane will be denoted in the sequel by $T_{z} Q \in G(d, k)$. We recall that $G(d, k)$ is the Grassmannian manifold of all $k$-dimensional linear subspaces of $\mathbb{R}^{d}$ and that the existence of a tangent $k$-plane $T_{z} Q$ for $Q$ at $z$ implies that, for every $0<s<1$,

$$
\lim _{r \rightarrow 0} \frac{1}{r^{k}} H^{k}\left(Q \cap B(z, r) \backslash Z\left(z, T_{z} Q, s\right)\right)=0,
$$

where

$$
Z(z, V, s)=\left\{x \in \mathbb{R}^{d}: \quad d(x-z, V)<s|x-z|\right\} .
$$

The space $G(d, k)$ has a natural measure $\gamma_{d, k}$ (see Section 3.9 in [Ma2] for its definition and basic properties). Given $V \in G(d, k)$ we denote by $P_{V}: \mathbb{R}^{d} \rightarrow V$ the orthogonal projection from $\mathbb{R}^{d}$ onto $V$. 
The following lemma is crucial since it gives rectifiability of the limit set provided the topological and Hausdorff dimensions coincide.

Lemma 2.1. If $S=\left\{\phi_{i}\right\}_{i \in I}$ is a conformal IFS and $H^{T D(\bar{J})}(\bar{J})=H^{T D(\bar{J})}(J)>0$, $H D(\bar{J})=T D(\bar{J})$, then the system $S$ is regular, $m=\left.\frac{H^{k}}{H^{k}(J)}\right|_{J}$ is the $k$-conformal measure on $J$ and the closure $\bar{J}$ is $T D(\bar{J})$-rectifiable.

Proof. Put $k=\operatorname{TD}(\bar{J})$. Since $\mathrm{H}^{k}(J)>0$ and since $\operatorname{HD}(J)=k$, we conclude from Theorem 4.16 in [MU1] that the system $S$ is regular and, using Lemma 4.2 in MU1, we deduce that $\mathrm{H}^{k}(J)<\infty$ and $m=\left.\frac{\mathrm{H}^{k}}{\mathrm{H}^{k}(J)}\right|_{J}$ is the $k$-conformal measure on $J$. Now, Federer's theorem on p. 545 in $[\mathrm{Fe}$ says that if the $s$-dimensional integral-geometric measure $\mathcal{I}_{1}^{s}(A)$ of a subset $A$ of a Euclidean space vanishes, then $\mathrm{TD}(A) \leq s-1$. Hence $\mathcal{I}_{1}^{k}(\bar{J})>0$. Since (see Ma2], p. 86)

$$
\mathcal{I}_{1}^{k}(\bar{J})=\int_{G(d, k)} \int_{V} \mathrm{H}^{0}\left(\bar{J} \cap \mathrm{P}_{V}^{-1}(a)\right) d \mathrm{H}^{k}(a) d \gamma_{d, k}(V),
$$

we therefore conclude that there exists a Borel set $G \subset G(d, k)$ with $\gamma_{d, k}(G)>0$ such that $\mathrm{H}^{0}\left(\bar{J} \cap \mathrm{P}_{V}^{-1}(a)\right)>0$ for every $V \in G$ and all $a$ in some set $W_{V} \subset V$ with $\mathrm{H}^{k}\left(W_{V}\right)>0$. In particular, $\mathrm{P}_{V}(\bar{J}) \supset W_{V}$ and therefore $\mathrm{H}^{k}\left(\mathrm{P}_{V}(\bar{J})\right)>0$ for all $V \in G$. Hence, it follows from Theorem 18.1(2) on p. 250 in Ma2] that $\bar{J}$ is not purely $k$-unrectifiable. Therefore, combining Theorems 17.6 (notice that although this is not indicated in Matillas's book, we need to know that $\mathrm{H}^{k}(\bar{J})>0$ for this theorem to actually make sense), Theorem 6.2(1) in [Ma2] and the fact that $\mathrm{H}^{k}(\bar{J})=\mathrm{H}^{k}(J)>0$, we conclude that $\Theta^{k}(\bar{J}, x)=1$ for all $x$ in some set $F \subset J$ with $\mathrm{H}^{k}(F)>0$, where the density functions $\Theta^{k}$ as well as $\Theta_{*}^{k}$ and $\Theta^{* k}$ were defined on p. 89 in [Ma2]. Now fix $x \in J$. It follows from an improved version of the distortion property (1d) that for all $i \in I$ and all $r>0$ small enough,

$$
\begin{aligned}
\mathrm{H}^{k}\left(\bar{J} \cap B\left(\phi_{i}(x),\left|\phi_{i}^{\prime}(x)\right| r\right)\right) & \geq \mathrm{H}^{k}\left(\phi_{i}\left(J \cap B\left(x, K_{r}^{-1} r\right)\right)\right) \\
& \geq K_{r}^{-k}\left|\phi_{i}^{\prime}(x)\right|^{h} \mathrm{H}^{k}\left(J \cap B\left(x, K_{r}^{-1} r\right)\right),
\end{aligned}
$$

where $K_{r}$ is such that $\lim _{r \rightarrow 0} K_{r}=1$. Hence

$$
\begin{aligned}
\frac{\mathrm{H}^{k}\left(\bar{J} \cap B\left(\phi_{i}(x),\left|\phi_{i}^{\prime}(x)\right| r\right)\right)}{\left(2\left|\phi_{i}^{\prime}(x)\right| r\right)^{k}} & \geq \frac{K_{r}^{-k}\left|\phi_{i}^{\prime}(x)\right|^{k}}{\left(2\left|\phi_{i}^{\prime}(x)\right| r\right)^{k}} \mathrm{H}^{k}\left(J \cap B\left(x, K_{r}^{-1} r\right)\right) \\
& =K_{r}^{-2 k} \frac{\mathrm{H}^{k}\left(J \cap B\left(x, K_{r}^{-1} r\right)\right)}{\left(2 K_{r}^{-1} r\right)^{k}},
\end{aligned}
$$

and letting $r \searrow 0$ we conclude that

$$
\Theta_{*}^{k}\left(\bar{J}, \phi_{i}(x)\right) \geq \Theta_{*}^{k}(\bar{J}, x) .
$$

Let $\tilde{m}$ be the lift of the conformal measure $m$ to the coding space $I^{\infty}$ and let $\tilde{\mu}$ be its shift-invariant version produced in Theorem 1.6. Since by this theorem the dynamical system $(\sigma, \tilde{\mu})$ is ergodic, it therefore follows from Birkhoff's Ergodic Theorem and (2.1) that the function $\omega \mapsto \Theta_{*}^{k}(\bar{J}, \pi(\omega))$ is constant $\tilde{\mu}$-a.e. Since $\tilde{\mu}\left(\pi^{-1}(F)\right)>0$, we therefore conclude that $\Theta_{*}^{k}(\bar{J}, \pi(\omega))=1$ for $\tilde{\mu}$-a.e. $\omega \in I^{\infty}$. Thus $\Theta_{*}^{k}(\bar{J}, x)=1$ for $\mathrm{H}^{k}$-a.e. $x \in \bar{J}$. Combining this with Theorem 6.2(1) in Ma2 we see that $\Theta^{k}(\bar{J}, x)$ exists and is equal to 1 for $\mathrm{H}^{k}$-a.e. $x \in \bar{J}$. Now invoking Theorem 17.6(1) in [Ma2] finishes the proof. 


\section{Proof of Theorem 1.2}

Put $k=\operatorname{TD}(\bar{J})$ and suppose that $\operatorname{HD}(J) \leq k$. Since $\operatorname{HD}(S(\infty))<\operatorname{HD}(J)$, using Proposition 1.1 we conclude that $\operatorname{HD}(\bar{J})=\operatorname{HD}(J) \leq k$. Hence, $\operatorname{HD}(\bar{J})=k$ and $\mathrm{H}^{k}(J)=\mathrm{H}^{k}(\bar{J})>0$, where this inequality follows from Marczewski's theorem (see Theorem vii 2, p. 104 in [HW]). Thus the assumptions of Lemma 2.1 are satisfied. In view of this lemma, the set $\bar{J}$ is $k$-rectifiable. By Theorem 15.19 in Ma2 this equivalently means that for $\mathrm{H}^{k}$-almost all $z \in J$ there is a unique approximate tangent $k$-plane $T_{z} \bar{J}$ for $\bar{J}$ at $z$.

We now fix such a point, say $z_{0}=\pi(\omega) \in J, \omega \in I^{\infty}$, and make the following renormalisation. Set $\lambda_{n}=\left|\phi_{\left.\omega\right|_{n}}^{\prime}\left(\pi\left(\sigma^{n}(\omega)\right)\right)\right|^{-1}$ and define then

$$
\beta_{n}(z)=\lambda_{n}\left(z-z_{0}\right) \text {. }
$$

It follows from the Bounded Distortion Property (1d) that each mapping $\beta_{n} \circ \phi_{\left.\omega\right|_{n}}$ : $X \rightarrow \mathbb{R}^{d}$ is locally Lipschitz continuous with the Lipschitz constant $K^{2}$ and from (1.2) that $\beta_{n} \circ \phi_{\left.\omega\right|_{n}}(X) \subset B(0, D K)$. Therefore, the Ascoli-Arzela theorem applies and there exists an increasing to infinity sequence of positive integers $\left\{n_{j}\right\}_{j=1}^{\infty}$ such that the sequence $\psi_{j}: X \rightarrow \mathbb{R}^{d}$ converges uniformly to a continuous function $\Psi: X \rightarrow \mathbb{R}^{d}$, where $\psi_{j}=\beta_{n_{j}} \circ \phi_{\left.\omega\right|_{n_{j}}}$. The limit function $\Psi: X \rightarrow X$ is conformal. We shall prove the following.

Claim 3.1. $\Psi(\bar{J}) \subset T_{z_{0}} \bar{J}$.

Proof. Suppose on the contrary that the claim does not hold. Then there exists an open bounded set $\Omega \subset \Psi(\bar{J})$ such that

$$
\eta=\operatorname{dist}\left(\Omega, T_{z_{0}} \bar{J}\right)>0 .
$$

Since $\Omega$ is an open subset of $\bar{J}$, we get $\mathrm{H}^{k}(\Omega)>0$. Put $U=\Psi^{-1}(\Omega)$ and $U_{j}=f_{j}(U)$. Then

$$
\begin{aligned}
0<\mathrm{H}^{k}(\Omega)=\int_{U}\left|\Psi^{\prime}\right|^{k} d \mathrm{H}^{k} & =\lim _{j \rightarrow \infty} \int_{U}\left|\psi_{j}^{\prime}\right|^{k} d \mathrm{H}^{k} \\
& =\lim _{j \rightarrow \infty} \mathrm{H}^{k}\left(\psi_{j}(U)\right)=\lim _{j \rightarrow \infty} \mathrm{H}^{k}\left(\beta_{n_{j}}\left(U_{j}\right)\right) .
\end{aligned}
$$

Hence, there exists $\tau>0$ and $j_{0} \geq 1$ such that

$$
0<\tau \leq \mathrm{H}^{k}\left(\beta_{n_{j}}\left(U_{j}\right)\right)=\lambda_{n_{j}}^{k} \mathrm{H}^{k}\left(U_{j}\right)
$$

for all $j \geq j_{0}$. Due to (3.1) and the boundednes of $\Omega$ we can choose $0<s<1$ such that $Z\left(0, T_{z_{0}} \bar{J}, 2 s\right) \cap \Omega=\emptyset$. Consider the cones $\mathcal{Z}=Z\left(0, T_{z_{0}} \bar{J}, s\right)$ and $\mathcal{Z}_{j}=$ $Z\left(z_{0}, T_{z_{0}} \bar{J}, s\right)=\beta_{n_{j}}^{-1}(\mathcal{Z})$. Fix also a ball $B=B(0, R)$ such that $\Omega \subset B(0, R / 2)$ and set $B_{j}=\beta_{n_{j}}^{-1}(B)=B\left(z_{0}, R \lambda_{n_{j}}^{-1}\right)$. Since $T_{z_{0}} \bar{J}$ is an approximate tangent $k$-plane of $\bar{J}$ at $z_{0}$, we have

$$
\lim _{j \rightarrow \infty} \lambda_{n_{j}}^{k} \mathrm{H}^{k}\left(\bar{J} \cap B_{j} \backslash \mathcal{Z}_{j}\right)=\lim _{j \rightarrow \infty}\left(R \lambda_{n_{j}}^{-1}\right)^{-k} \mathrm{H}^{k}\left(\bar{J} \cap B_{j} \backslash \mathcal{Z}_{j}\right)=0 .
$$

But, if $j$ is sufficiently large, then $\beta_{n_{j}}\left(U_{j}\right)=\Psi_{j} \circ \Psi^{-1}(\Omega) \subset B \backslash \mathcal{Z}$ and therefore $U_{j} \subset \bar{J} \cap B_{j} \backslash \mathcal{Z}_{j}$. It then follows from (3.2) that

$$
\lambda_{n_{j}}^{k} \mathrm{H}^{k}\left(\bar{J} \cap B_{j} \backslash \mathcal{Z}_{j}\right) \geq \lambda_{n_{j}}^{k} \mathrm{H}^{k}\left(U_{j}\right) \geq \tau>0,
$$


and this contradicts (3.3). We thus proved the claim and therefore the "smooth or fractal" dichotomy announced in Theorem 1.2

We are left to show that if one of the maps $\phi_{i}$ is a similarity map $\left(\lambda_{i} A_{i}+a_{i}, 0<\right.$ $\left.\lambda_{i}<1\right)$, then $\bar{J}$ is contained in a $k$-dimensional hyperspace of $\mathbb{R}^{d}$. Indeed, suppose on the contrary that $\bar{J} \subset Q$, a geometric sphere in $\mathbb{R}^{d}$. Since $\phi_{i}(Q)=\lambda_{i} A_{i}(Q)+a_{i}$ is a geometric sphere of dimension $k$ and the sphere $Q \cap \phi_{i}(Q)$ contains the $k$ dimensional set $\bar{J}$, this intersection is a $k$-dimensional sphere, and therefore equal to both $Q$ and $\phi_{i}(Q)$. This contradicts the fact that $\phi_{i}: Q \rightarrow Q$ is a strict contraction with a Lipschitz constant equal to $\lambda_{i}$. We are done.

In the case of a conformal expanding repeller $F$ appearing in Theorem 1.5 the proof of Lemma 2.1 requires only minor obvious modifications. In the proof of Claim 3.1 one replaces $X$ by balls of some sufficiently small radii and the maps $\phi_{\omega_{n_{j}}}$ by appropriate inverse branches of forward iterates of $F$. We thus get that each point of $Y$ has a neighbourhood that is an open subset of either a $k$-dimensional geometric sphere or hyperspace. Compactness of $Y$ proves the first part of the theorem. If $Y$ is connected this finite union clearly reduces to a one-sphere or a hyperspace.

\section{REFERENCES}

[BP] R. Benedetti and C. Petronio, Lectures on Hyperbolic Geometry, Springer-Verlag, Berlin, 1992. MR 94e:57015

[Bo] R. Bowen, Hausdorff dimension of quasi-circles, Inst. Hautes Études Sci. Publ. Math. 50 (1979), 11-25. MR 81g:57023

[Fe] H. Federer, Dimension and measure, Trans. Amer. Math. Soc. 62 (1947), 536-547. MR 9:339g

[HW] W. Hurewicz and H. Wallman, Dimension Theory, Princeton University Press, 1941. MR 3:312b

[Ma1] P. Mattila, On the structure of self-similar fractals, Ann. Acad. Sci. Fenn. Ser. A I Math. 7 (1982), 189-192. MR 84j:28011

[Ma2] P. Mattila, Geometry of sets and measures in Euclidean spaces. Fractals and rectifiability, Cambridge University Press, 1995. MR 96h:28006

[MMU] D. Mauldin, V. Mayer, and M. Urbański, Rigidity of connected limit sets of conformal IFSs, Michigan Math. J. 49 (2001), 451-458. MR 2002j:37057

[MU1] R. D. Mauldin and M. Urbański, Dimensions and measures in infinite iterated function systems, Proc. London Math. Soc. (3) 73 (1996), 105-154. MR 97c:28020

[MU2] R. D. Mauldin and M. Urbański, Conformal repellors with dimension one are Jordan curves, Pacific Journal of Math. 166 (1994), 85-97. MR 95k:58099

[Pr] F. Przytycki, On holomorphic perturbations of $z \rightarrow z^{n}$, Bull. Polish Acad. Sci. Math. 34 (1986), 127-132. MR 88a:58108

[PUZ] F. Przytycki, M. Urbański, and A. Zdunik, Harmonic, Gibbs and Hausdorff measures on repellers for holomorphic maps, I, Ann. Math. 130 (1989), 1-40. MR 91i:58115

$[\mathrm{Ru}] \quad$ D. Ruelle, Repellers for real analytic maps, Ergodic Theory and Dynam. Syst. 2 (1982), 99-107. MR 84f:58095

[Su] D. Sullivan, Seminar on conformal and hyperbolic geometry by D. P. Sullivan (Notes by M. Baker and J. Seade), preprint IHES (1982).

[U1] M. Urbański, On the Hausdorff dimension of a Julia set with a rationally indifferent periodic point, Studia Math. 97 (1991), 167-188. MR 93a:58146

[U2] M. Urbański, Hausdorff measures versus equilibrium states of conformal infinite iterated function systems, Periodica Math. Hungar., 37 (1998), 153-205. MR 2001k:28012

[U3] M. Urbański, Rigidity of multi-dimensional conformal iterated function systems, Nonlinearity 14 (2001), 1593-1610. MR 2003c:37031

[UV] M. Urbański and A. Volberg, A rigidity theorem in complex dynamics, in Fractal Geometry and Stochastics, Progress in Probability 37, Birkhäuser-Verlag (1995). MR 98b:58144 
[Va] J. Väisälä, Lectures on n-dimensional quasiconformal mappings, Lecture Notes in Math. 229, Springer-Verlag, 1971. MR 56:12260

[Z1] A. Zdunik, Parabolic orbifolds and the dimension of the maximal measure for rational maps, Invent. Math. 99 (1990), 627-649. MR 90m:58120

[Z2] A. Zdunik, Harmonic measure versus Hausdorff measures on repellors for holomorphic maps, Trans. Amer. Math. Soc. 326 (1991), 633-652. MR 91k:58071

Université de Lille I, UFR de Mathématiques, UMR 8524 du CNRS, 59655 Villeneuve D'Ascq Cedex, France

E-mail address: volker.mayer@univ-lille1.fr

$U R L$ : http://www-gat.univ-lille1.fr/ mayer

Department of Mathematics, University of North Texas, P.O. Box 311430, Denton, Texas 76203-1430

E-mail address: urbanski@unt.edu

$U R L$ : http://www . math. unt. edu/ urbanski 